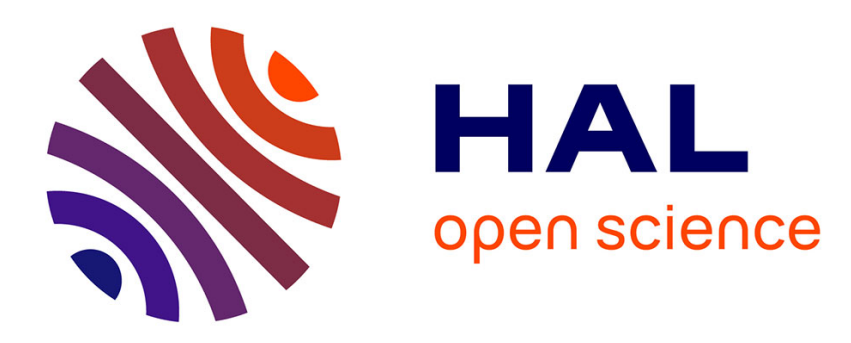

\title{
La route (libertarienne) de la servitude (transhumaniste)
} Sébastien Caré

\section{To cite this version:}

Sébastien Caré. La route (libertarienne) de la servitude (transhumaniste). Raisons politiques, 2019, 2019/2 (74), pp.13-28. 10.3917/rai.074.0013 . hal-02474524

\section{HAL Id: hal-02474524 https://hal-univ-rennes1.archives-ouvertes.fr/hal-02474524}

Submitted on 11 Feb 2020

HAL is a multi-disciplinary open access archive for the deposit and dissemination of scientific research documents, whether they are published or not. The documents may come from teaching and research institutions in France or abroad, or from public or private research centers.
L'archive ouverte pluridisciplinaire HAL, est destinée au dépôt et à la diffusion de documents scientifiques de niveau recherche, publiés ou non, émanant des établissements d'enseignement et de recherche français ou étrangers, des laboratoires publics ou privés. 


\section{La route (libertarienne) de la servitude (transhumaniste)}

SÉBASTIEN CARÉ

Le transhumanisme et le libertarianisme sont encore aujourd'hui fréquemment apparentés, sinon parfois confondus ${ }^{1}$. L'affiliation a tout d'abord des raisons historiques, les premières boutures du transhumanisme contemporain ayant été développées au début des années 1990 par des penseurs ne faisant pas mystère de leurs penchants libertariens ${ }^{2}$. Ainsi par exemple des extropiens qui, sous la houlette de Max More, n’ont jamais dissimulé leur sympathie pour les pensées libertarienne ou anarcho-capitaliste, et ont longtemps dominé la mouvance transhumaniste. Mais on peut aussi évoquer des raisons plus profondes, et repérer entre les deux théories une forme d'affinité élective ${ }^{3}$ établissant un rapport de convergence et d'emprunts réciproques autour de principes mutuellement partagés comme la propriété sur soi, le libre consentement, le choix laissé à chacun de faire ce qu’il souhaite de son propre corps ou encore une certaine méfiance manifestée à l'endroit d'une autorité étatique soupçonnée de vouloir indûment s’immiscer dans les plans de vie individuels. La congruence entre les deux doctrines est aujourd'hui telle que les tentatives d'amarrer le transhumanisme à d'autres quais politiques sont aussitôt suspectées de véhiculer en contrebande un libertarianisme qui ne dirait pas son nom.

Cet article aimerait interroger le sens et les implications de ce lien historiquement constitué, et aujourd'hui encore empiriquement observable ${ }^{4}$, entre libertarianisme et

${ }^{1}$ Cette affiliation est notamment défendue par certains de ses partisans, qui prônent un « transhumanisme libertarien », ou un «techno-libertarianisme ». Ainsi de l'éditorialiste du magazine libertarien Reason Ronald Bailey (Liberation Biology: The Scientific And Moral Case For The Biotech Revolution, Amherst, Prometheus Books, 2005), du professeur de droit et blogger Glenn Reynolds (Glenn Reynolds, An Army of Davids: How Markets and Technology Empower Ordinary People to Beat Big Media, Big Government, and Other Goliaths, Nashville, Thomas Nelson, 2006) ou, plus récemment, de l'éditorialiste du Cato Unbound Jason Kuznicki, (Jason Kuznicki, Technology and the End of Authority. What Is Government For?, Basingstoke, Palgrave Macmillan, 2017).

${ }^{2}$ Sur l'histoire de ces liens, voir notamment les travaux pionniers de Richard Barbrook et Andy Cameron (Richard Barbrook et Andy Cameron, «The Californian Ideology », Mute, Volume 1, Numéro 3, 1995) et Paulina Borsook, Cyberselfish : A Critical Romp through the Terribly Libertarian Culture of High Tech, New York, PublicAffairs, 2000, p. 73-114.

${ }^{3}$ En s'appuyant sur les rares occurrences du syntagme dans l'œuvre de Max Weber, Michael Lowy définit l' «affinité élective » comme «le processus par lequel deux formes culturelles - religieuses, intellectuelles, politiques ou économiques - entrent, à partir de certaines analogies significatives, parentés intimes ou affinités de sens, dans un rapport d'attraction et influence réciproques, choix mutuel, convergence active et renforcement mutuel. » Michael Löwy, «Le concept d'affinité élective chez Max Weber », Archives de sciences sociales des religions, Numéro 127, 2004, p. 100.

${ }^{4}$ Bien que l'Extropy Institute ait été fermé en 2006, le sociologue transhumaniste progressiste James Hughes considère par exemple que les libertariens occupent encore une place importante dans le mouvement transhumaniste contemporain à travers le «singularisme » défendue par Ray Kurzweil, Hugo de Garis, Hans 
transhumanisme. Pour ce faire, il nous faut au préalable nous entendre sur les définitions qu'il convient de donner aux deux termes, qui ont en commun de rassembler des tendances très diverses. Nous pouvons de manière économe définir négativement le libertarianisme comme une théorie politique minimale refusant que l'État se mêle d'autres choses que la sauvegarde de la sécurité de ses administrés. Les libertariens plaident ainsi pour un retrait significatif de l'autorité publique, non seulement dans le commerce des individus, mais aussi dans la manière dont ils conduisent leur vie, et joignent par exemple à la dérèglementation du marché la légalisation des drogues, au démantèlement de l’État providence la défense d'une liberté d'expression absolue ou encore à la lutte contre l'impérialisme américain la justification du droit de porter une arme. Le libertarianisme se distingue par son ambition de projeter la logique du marché sur toutes les sphères de la vie sociale, et d'ainsi sortir le libéralisme du seul domaine économique dans lequel il avait tendance à se cloîtrer. Le transhumanisme se présente quant à lui comme « le mouvement intellectuel et culturel qui affirme la possibilité et la désirabilité d'une amélioration fondamentale de la condition humaine par la raison appliquée, notamment en développant et en rendant largement accessibles les technologies qui éliminent l'âge et augmentent les capacités humaines intellectuelles, physiques et psychologiques $^{5}$. » L'examen des liens entre libertarianisme et transhumanisme se heurte à cette difficulté qui est que chacun des deux termes peut être dédoublé et désigner à la fois une réalité empirique possible (voire en voie de concrétisation) et la théorie normative venant la justifier pour hâter son avènement ${ }^{6}$. Deux manières d'interroger le lien sont alors envisageables. La première, qui occupera les trois premières parties de cette contribution, consiste à voir si les deux propositions théoriques s’impliquent réciproquement. Autrement dit: le transhumanisme est-il nécessairement libertarien? Le libertarianisme est-il nécessairement transhumaniste? Après avoir répondu négativement à chacune des deux

Moravec et Peter Thiel. Hughes considère même que, depuis 2019, « les libertariens et les singularistes, soutenus par la philanthropie de Thiel, ont étendu leur hégémonie sur la communauté transhumaniste » (James Hughes, « The Politics of Transhumanism and the Techno-millennial Imagination (1626-20130) » Zygon, Volume 47, Numéro 4, Décembre 2012, p. 766).

5 «What is Transhumanism?» [http://whatistranshumanism.org/]. Cette définition, fréquemment citée, a l'avantage de laisser ouverte la question de l'affiliation politique du transhumanisme, en ne précisant pas qui, du marché et de l'État, aurait la charge d’étendre l'accessibilité aux technologies évoquées.

${ }^{6}$ On notera ici une certaine asymétrie entre les deux. En tant que réalité en devenir, le transhumanisme se révèle en un sens plus avancé que le libertarianisme. Pour le dire de manière schématique: les technologies amélioratives progressent davantage que le retrait de l’État. En revanche, la théorie libertarienne, systématisée dans les années 1970 et aujourd'hui développée par certains universitaires, paraît beaucoup plus élaborée que la doctrine transhumaniste, encore balbutiante, et davantage portée par des entrepreneurs praticiens peu rigoureux que par des intellectuels de métier. Francis Fukuyama nous semble ainsi faire fausse route lorsqu'il décrit le transhumanisme comme «l'idée la plus dangereuse au monde » (Francis Fukuyama, «Transhumanism: The World' Most Dangerous Idea », Foreign Policy, Numéro 144, 2004, p. 42-43). L’idéologie du transhumanisme se révèle à l'examen assez fragile et peu crédible. En revanche, la réalité qu'elle appelle de ses vœux, ou celle advenant qu'elle justifie, semble de plus en plus irrésistible. 
questions (I), nous explorerons plus spécifiquement l'implication qui voudrait que le libertarianisme soit nécessairement transhumaniste, en examinant successivement les pensées de Ayn Rand (II) et de Friedrich Hayek (III), auxquelles les transhumanistes se réfèrent allègrement, mais d'une manière parfois tronquée trahissant une affinité très sélective. La dernière partie (IV) interrogera les rapports entre transhumanisme et libertarianisme d'une manière différente, en s'efforçant d'envisager la compossibilité de leurs réalisations respectives. Nous montrerons alors que, si le libertarianisme n’apporte aucune justification théorique au mode de vie promu par le transhumanisme, il ne le proscrit pas, et sa concrétisation (qu'attesterait un retrait significatif de l'État) viendrait même l'encourager en instaurant une concurrence exacerbée et en créant ainsi faisant un besoin de performance que les technologies amélioratives voudraient pouvoir satisfaire. Nous conclurons cependant qu'en retour, la réalisation du transhumanisme, et l'avènement de l'homme augmenté, consacrerait l'obsolescence d'une théorie libertarienne originellement destinée à des êtres très imparfaits, et ouvrirait la possibilité de son contraire totalitaire.

\section{L’absence d'implication réciproque entre transhumanisme et libertarianisme}

On peut tout d'abord tenter d'interroger le lien entre les deux théories en termes d'équivalence logique et voir si elles s’impliquent réciproquement. Deux propositions doivent alors être examinées. La première consiste à dire que le transhumanisme ne peut pas ne pas être libertarien, autrement dit que l'appel à l'avènement d'un homme nouveau, augmenté par la technique, passe nécessairement par la défense d'un État minimal, voire l'adoption d'une position anarcho-capitaliste. La seconde proposition énoncerait réciproquement que le libertarianisme est nécessairement transhumaniste : le rejet d’un État étendant ses prérogatives au-delà de la simple protection des personnes supposerait une amélioration fondamentale de la condition humaine et la promotion de toutes les technologies susceptibles d'y conduire.

L’histoire du transhumanisme évoquée précédemment rend plausible l’implication initiale, pour laquelle viennent aussi plaider de nombreuses résonnances entre les thèses fréquemment défendues par les deux courants. Ainsi par exemple du principe de propriété sur soi qui offre à chacun une liberté absolue sur son propre corps, et interdit à l'État de s’immiscer dans les choix individuels et d'ainsi prohiber les conduites et les techniques encouragées par les transhumanistes. Cette association nous semble pourtant hautement 
circonstancielle, et dépendante d'un contexte où l'autorité étatique envisage de restreindre l’accès aux technologies amélioratives. Il suffit ainsi de considérer la situation, tout à fait possible, dans laquelle un État partagerait l'ambition transhumaniste de faire advenir un nouvel homme, et d’ainsi promouvoir en les finançant les techniques permettant d'améliorer chaque individu, pour comprendre que le transhumaniste pourrait très bien changer de crèmerie politique et ne pas être libertarien. Pour s'en convaincre, on peut tout d'abord rappeler que, si les transhumanistes, à l'instar de Max More, se sont longtemps massivement réclamés de la tradition libertarienne, ils sont de plus en plus nombreux aujourd'hui à revendiquer une tout autre allégeance doctrinale. L’exemple sans doute le plus significatif de cette ouverture à d'autres théories politiques est l'ambition nourrie par James Hughes d'offrir une troisième voie progressiste, qu’il qualifie de «transhumanisme démocratique », et qui dépasserait l'opposition entre luddites bioconservateurs et libertariens technophiles ${ }^{7}$. Si l'on peut évidemment émettre des doutes sur la cohérence d'un tel positionnement ${ }^{8}$, la défense d'un transhumanisme qui ne serait pas libertarien nous paraît cependant tout à fait possible. On pourrait par exemple parfaitement envisager une théorie égalitariste qui, dans le sillage de John Rawls ${ }^{9}$, verrait dans l'amélioration technique de la condition humaine un moyen d'égaliser la distribution des biens premiers naturels inaliénables ${ }^{10}$. Certains vont même jusqu'à vouloir établir des liens avec le marxisme ${ }^{11}$, et la perspective d'un totalitarisme transhumaniste, où l’État réduirait les hommes à n’être que des machines, n’est pas en soi contradictoire $^{12}$.

\footnotetext{
${ }^{7}$ James Hughes, Citizen Cyborg. Why Democratic Societies Must Respond to the Redesigned Human of the Future, Cambridge, Westview Press, 2004. Voir aussi James Hughes, «The Politics of Transhumanism and the Techno-millennial Imagination (1626-20130) », art. cité, p. 757-776.

${ }^{8}$ Voir par exemple l'article de Stanislas Deprez dans ce numéro.

${ }^{9}$ Il est à noter que Rawls admettait lui-même à cette fin, quoique du bout des lèvres, la possible légitimité d'une politique eugéniste. «Il est possible d'adopter une politique d’eugénisme, plus ou moins explicitement. [...] Une société doit prendre des dispositions pour, au moins, préserver le niveau général des capacités naturelles et pour empêcher la diffusion de défauts graves. [...] Nous pouvons supposer qu'à long terme, s’il y a une limite supérieure aux capacités, nous arriverons finalement à une société où règne la plus grande liberté égale pour tous et dont les membres jouissent de la plus grande égalité de talents. » John Rawls, Théorie de la justice (1971), traduit de l'américain par Catherine Audard, Paris, Seuil, 1997 (1987), p. 138.

${ }^{10}$ C'est dans une telle perspective que certains ont récemment justifié le recours à une forme libérale d’eugénisme. Voir A. Buchanan, D. W. Brock, N. Daniels, D. Wikler, From Chance to Choice. Genetics and Justice, Cambridge, Cambridge University Press, 2000. «L’intervention génétique sera un moyen important de réaliser l’égalité des chances », Ibid., p. 16.

11 James Steinhoff, «Transhumanism and Marxism: Philosophical Connections », Journal of Evolution and Technology, Volume 24, Numéro 2, May 2014, p. 1-16.

${ }^{12}$ Gilbert Hottois a tort de prétendre que le transhumanisme serait «viscéralement hostile à toute forme de totalitarisme : le monde d'Aldous Huxley et celui de George Orwell sont radicalement anti-transhumanistes. » Gilbert Hottois, Philosophie et idéologies trans/posthumanistes, Paris, Vrin, 2017, p. 157. C'est d'ailleurs pour prévenir le risque d'une telle association que Ronald Bailey justifie sa propre adhésion au libertarianisme, sans lequel « on ne pourrait résister à la tentation d'imposer démocratiquement des améliorations, ce qui consisterait à imposer à ceux qui n’en veulent pas une vision particulière de l'épanouissement humain » (Ronald Bailey, « For
} 
Statuer sur la validité de l’implication réciproque, selon laquelle le libertarianisme ne pourrait être que transhumaniste, semble à première vue encore plus délicat, notamment parce que le libertarianisme a été systématisé (au milieu des années 1970) bien avant la formation du courant transhumaniste, et qu'aucun de ses auteurs canoniques n'a explicitement pris position sur le sujet. La seule présence, dans le mouvement libertarien, d’auteurs ouvertement conservateurs, hostiles à l'avortement et à la thérapie génique, rassemblés sous la bannière de paléo-libertariens, pourrait toutefois suffire à infirmer l'hypothèse d'un lien nécessaire. Mais il y a plus : au moins deux éléments fondamentaux et récurrents dans la théorie libertarienne viennent contrarier l'idée d'un possible recoupement entre les deux points de vue. Le premier est que le libertarianisme ne se présente le plus souvent que comme une théorie politique, qui vise à déterminer les conditions sociales d'une vie pacifique entre des hommes poursuivant des conceptions diverses et irréconciliables du bien, et qui se refuse ainsi à aborder la question des comportements moraux individuels au sujet desquels le transhumanisme n'est pas, quant à lui, sans formuler quelques hypothèses. Là où le libertarianisme ne serait qu'une doctrine minimaliste se gardant de définir les principes d'une vie vertueuse, le transhumanisme porterait un projet maximaliste ${ }^{13}$ envisageant des devoirs envers soi-même (notamment celui de s'améliorer) et indiquant la voie d'un accomplissement humain particulier: il est une doctrine compréhensive assimilable à une religion. Si les deux ambitions théoriques ne sont pas incompatibles, elles demeurent ainsi bien distinctes. Le second élément, qui découle du précédent, est que le libertarianisme, à la différence du transhumanisme, envisage l’homme tel qu'il est, et non tel qu'il voudrait qu'il fût, et se trouve ainsi conduit à s’intéresser aux conditions sociales de coexistence d'hommes imparfaits, limités et vulnérables. Ces deux éléments nous invitent à interroger la légitimité des emprunts couramment faits par les transhumanistes à certaines idées libertariennes. Nous privilégierons alors les deux références qui nous semblent les plus souvent revendiquées par les libertariens transhumanistes : Ayn Rand et Friedrich Hayek.

\section{Le héros randien contre le super héros transhumaniste}

Enhancing People », in Max More \& Natasha Vita-More (dir.), The Transhumanist Reader. Classical and Contemporary Essays on the Science, Technology, and Philosophy of the Human Future, Chichester, WileyBlackwell, 2013, p. 339. Pour Bailey, un totalitarisme transhumaniste serait certes un danger, mais n'est aucunement une impossibilité logique.

${ }^{13} \mathrm{La}$ distinction entre maximalisme et minimalisme est empruntée à Ruwen Ogien. R. Ogien, L'éthique aujourd'hui : maximalistes et minimalistes, Paris, coll. « Folio Essais », Gallimard, 2007. 
Parmi les auteurs libertariens dont les transhumanistes se réclament, Ayn Rand est certainement la plus fréquemment citée ${ }^{14}$. Cela s'explique par le fait que la romancière, à l'instar des transhumanistes, mais à la différence des autres libertariens, adopte elle-aussi clairement une éthique maximaliste définissant les principes d'une morale individuelle encourageant l'égoïsme et vantant le recours à la technique ${ }^{15}$. Parmi les avancées technologiques promues par les transhumanistes, l'exigence de la morale randienne implique toutefois de faire le tri, et une lecture plus attentive de son œuvre devrait décourager chez eux des emprunts peu scrupuleux. Il faut en effet rappeler que Rand revendique une éthique aristotélicienne, ce qui implique au moins deux idées peu compatibles avec le transhumanisme. La première est que c'est bien la nature, et non son dépassement, qui doit constituer la norme. « Le fait qu'une entité vivante est détermine ce qu'elle devrait faire ${ }^{16}$ » : «Pour tout organisme conscient, pour toute conscience vivante, la connaissance est le moyen de survie, et chaque "est" implique un "doit" ${ }^{17}$. » Contrairement aux transhumanistes, Ayn Rand n’entend pas soustraire l'être humain aux impératifs de la nature. Il en résulte chez elle une certaine confiance en la nature humaine, qui contraste avec la vision dépréciative que s’en font les transhumanistes pour lesquels l'humain ne serait qu'un être chétif, vulnérable qu'il s’agirait de réparer ${ }^{18}$. La deuxième idée empruntée par Rand à Aristote est que la vertu est appréhendée en termes de dispositions acquises, qui doivent ainsi être cultivées et qui ne peuvent s'obtenir qu'au prix d'un effort dont certains transhumanistes aimeraient pouvoir exempter les individus. Pour Rand en effet, « puisque tout ce dont l'homme a besoin doit être découvert par son propre esprit et produit par son propre effort, les deux aspects essentiels de

${ }^{14}$ Pour une analyse approfondie des liens entre l’objectivisme randien et les idées transhumanistes, voir Ben Murnane, Ayn Rand and the Posthuman. The Mind-Made Future, Londres, Palgrave Macmillan, 2018, p. 55-91.

15 « Il est intéressant d'analyser les raisons qui rendent les hommes si désireux de s'abaisser eux-mêmes. Ce besoin qu'ils ont par exemple de se sentir petits devant la nature. [...] Avez-vous remarqué l'air vertueux qu'un homme prend lorsqu'il exprime de tels sentiments. Regardez-moi, a-t-il l'air de dire, je suis si fier de me sentir un pygmée, je me sens tellement vertueux ! N'avez-vous jamais remarqué avec quelles délices les gens citent les paroles de quelque célébrité déclarant qu'elle se sent bien peu de chose lorsqu'elle est devant les chutes du Niagara. [...] Mais ce ne sont certes pas ces gens-là qui ont enchaîné le feu, l'eau et l'électricité, traversé l'océan en sloop, construit des avions, des barrages... des gratte-ciel. Qu'est-ce donc dont ils ont si peur et qu'ils haïssent tant, ceux qui aiment à se vautrer dans la boue, et quelle joie y trouvent-ils ? » Ayn Rand, La Source Vive (1943), traduit de l'américain par Jane Fillion, Paris, Plon, 1997, p. 442.

${ }^{16}$ Ayn Rand, La vertu d'égoïsme (1964), traduit de l’américain par Marc Meunier Paris, Les Belles Lettres, « Laissez-faire », 1993, p. 32.

${ }^{17}$ Ibid., p. 49.

${ }^{18}$ Voir la lettre adressée par Max More à « Mère Nature » : « Sans vouloir te manquer de respect, concernant la constitution humaine, tu n'as pas toujours bien travaillé. Tu nous as faits vulnérables aux maladies et aux blessures. Tu nous obliges à vieillir et à mourir, juste au moment où nous commençons à atteindre la sagesse. [...] Tu nous as équipés d'une mémoire limitée, de très peu de capacité de contrôle, d'impulsions tribales et xénophobes. Et tu as oublié de nous transmettre le mode d'emploi de notre fonctionnement ! » Max More, «A letter to Mother Nature », cité par Nicolas Le Dévédec, La société de l'amélioration. La perfectibilité humaine des Lumières au transhumanisme, Montréal, Liber, 2015, p. 204-205. 
la méthode de survie qui convient à un être rationnel sont la réflexion et le travail productif $^{19}$.» Or, il est à craindre que le transhumanisme réalisé et l'avènement du posthumain dispensent ultimement l'humain d'une telle réflexion et d'un tel travail productif, sans lesquels aucune vie morale n'est pour Rand possible.

Le transhumanisme va ainsi à l'encontre de l'«ambition morale » d'Ayn Rand, qui entend faire de chaque individu « un self-made-man dans le domaine spirituel ${ }^{20}$ ». Le projet transhumaniste est bien différent, qui vise en fin de compte à permettre à chacun, ou du moins à ceux qui le veulent et aux rares qui le peuvent, de profiter de la science des autres. Le posthumain serait à l'opposé du self-made-man; il serait un cyborg essentiellement fait par d'autres. Le rêve nourri par certains transhumanistes ${ }^{21}$ d'une numérisation intégrale de la personnalité aboutirait par exemple à une dissolution de l'individualité. Il est ici intéressant de noter que Jürgen Habermas, pourtant identifié comme un bioconservateur, emploie un argument semblable à l'encontre de ce qu'il nomme l'«eugénisme libéral », qui aurait l'inconvénient de violer l'autonomie de l’individu programmé, soumis malgré lui «aux intentions fixées par un tiers » et réduit à ne pouvoir prétendre être «l'auteur sans partage de sa vie personnelle ${ }^{22}$. Habermas défend ici un argumentaire kantien, qui permet de décrire les individus augmentés comme des mineurs sous tutelle ${ }^{23}$. Le cyborg posthumain serait ainsi un être en état de minorité, pouvant télécharger dans son cerveau une encyclopédie sans se donner la peine de la lire, susceptible d'accéder à la vie éternelle sans avoir à se donner les moyens de gagner le ciel, et capable de se maintenir en bonne santé sans devoir se soumettre au moindre régime ${ }^{24}$. Au fond, l'écrasante majorité des posthumains ne seraient selon les

\footnotetext{
${ }^{19}$ Ayn Rand, La vertu d'égoïsme, op. cit., p. 52.

${ }^{20}$ Ibid., p. 64.

${ }^{21}$ Voir par exemple le projet Neuralink d'Elon Musk. Pour Rand, «la connaissance n'arrive pas à l'homme d'une manière automatique » (Ibid., p. 67).

22 Jurgen Habermas, L'avenir de la nature humaine. Vers un eugénisme libéral ?, traduit de l'allemand par Christian Bouchindhomme, Paris, Gallimard, 2002, p. 96.

23 « La paresse et la lâcheté sont les causes qui font qu’un aussi grand nombre d’hommes préfèrent rester mineurs leur vie durant, longtemps après que la nature les a affranchis de toute direction étrangère ; et ces mêmes causes font qu'il devient si facile à d'autres de se prétendre leurs tuteurs. Il est si aisé d'être mineur ! Avec un livre qui tient lieu d'entendement, un directeur de conscience qui me tient lieu de conscience, un médecin qui juge pour moi de mon régime, etc., je n’ai vraiment pas besoin de me donner moi-même de la peine. Il ne m'est pas nécessaire de penser, pourvu que je puisse payer ; d'autres se chargeront bien pour moi de cette ennuyeuse besogne. » Emmanuel Kant, Qu'est-ce que les Lumières ? (1784), traduit de l'allemand par JeanMichel Muglioni, Paris, Hatier, 2005, p. 4.

${ }^{24}$ Nick Bostrom écrit ainsi, avec une franchise déconcertante : «Les médicaments et la thérapie génique vont conduire à une facette multidimensionnelle du bien-être. Ils pourront aussi bien modifier la personnalité qu'aider à surmonter la timidité, vaincre la jalousie, accroître la créativité et augmenter la capacité d'empathie envers les autres ainsi que la profondeur émotionnelle. Pensez aux prières, aux jeunes et à la discipline de fer auquel les gens se sont astreints au cours des siècles dans le but d'ennoblir leur personnalité. Bientôt, il sera possible d'atteindre le même but et de manière plus absolue en prenant quotidiennement un simple mélange de
} 
mots de Rand que des «parasites mentaux »: «Bien que certains hommes choisissent de ne pas utiliser leur faculté rationnelle, mais survivent quand même en imitant et répétant, comme des animaux savants, les sons et gestes routiniers qu'ils ont appris des autres, sans jamais faire l'effort de comprendre ce qu'ils font, le fait demeure que leur survie n'est rendue possible que grâce à ceux qui ont choisi d’utiliser leur faculté rationnelle et de découvrir les choses que leurs imitateurs ne font que répéter. La survie de tels parasites mentaux est purement aléatoire ; leur esprit non concentré est incapable de savoir qui imiter et quoi faire. Ce sont eux qui s'enfoncent dans l'abîme, à la remorque de n'importe quel tyran qui leur promet d'assumer la responsabilité d'être conscient ${ }^{25}$. » Ayn Rand emploie ainsi à l'encontre de ceux dont la survie dépendrait du pouvoir et du savoir des autres des mots très sévères, et qui pourraient très bien être adressés aux transhumanistes les plus zélés : « De la même façon que l'homme est libre de tenter de survivre n'importe comment, comme un parasite, un mendiant ou un pilleur, mais n'est pas libre d'y réussir autrement que provisoirement, il est libre de chercher son bonheur dans toutes sortes de fraudes irrationnelles, de caprices, d'illusions ou de stupides évasions de la réalité, mais pas libre d’y réussir autrement que provisoirement, ni d’échapper aux conséquences ${ }^{26}$. »

Le plaidoyer randien en faveur du progrès technique ne saurait donc tromper: il vise moins au fond une adaptation de l'homme à son environnement qu'une adaptation de l'environnement à l'homme. Ayn Rand écrit explicitement que «le travail productif est [...] le processus qui libère l'homme de la nécessité de s’adapter à son environnement, comme le font les animaux, et lui donne le pouvoir d'adapter son environnement à lui-même ${ }^{27}$. " Dans Citizen Cyborg, James Hughes donne ainsi un exemple qui trahit l'ambition transhumaniste, à rebours du projet randien. Pour lui, l’obésité ne viendrait que du hiatus entre nos modes de vie sédentaire et les caractéristiques de nos organismes, semblables à ceux de nos ancêtres chasseurs-cueilleurs, et destinés à emmagasiner d'importantes réserves nutritionnelles pour supporter les efforts nécessaires. «La cause première de l’obésité est que nous avons des corps conçus pour passer des heures à marcher chaque jour dans la savane, et des cerveaux qui trouvent les moyens d'accéder facilement aux graisses, sucres et glucides irrésistibles. Seules des thérapies génétiques et pharmaceutiques sures et accessibles peuvent mettre un

comprimés. » Nick Bostrom, «Qu’est-ce que le transhumanisme », cité dans Nicolas Le Dévédec, La société de l'amélioration, op. cit., p. 209.

${ }^{25}$ Ibid., p. 52-53.

${ }^{26}$ Ibid., p. 70-71.

${ }^{27}$ Ibid., p. 63. 
terme à cette funeste montée de l'obésité à travers le monde ${ }^{28}$. » Le plus sûr moyen de maigrir ne serait donc pas de manger moins ou de faire du sport, mais d'ingurgiter des pilules mangeuses de graisse. Rand vante davantage la capacité de l'homme à transformer le monde extérieur que sa capacité à se transformer lui-même ${ }^{29}$. Il y a chez Rand un éloge de l'authenticité, de la constance, de l'aptitude de l'homme à demeurer toujours le même et à ne pas se dérober, à la première occasion, aux fins que lui impose sa propre nature. Ayn Rand ambitionne de faire de chaque homme un héros, mais pas un super-héros doté de pouvoirs surnaturels. L'avènement du posthumanisme rendrait impraticable la morale randienne.

\section{Le libertarianisme hayékien : une théorie politique destinée à l'homme imparfait}

Le deuxième auteur libertarien, après Ayn Rand, à être régulièrement cité par les transhumanistes est Friedrich Hayek. Dans l'exposé de sa pensée extropienne, Max More fait par exemple du concept hayékien $d^{\text {' } « ~ o r d r e ~ s p o n t a n e ́ ~ » ~ l e ~ c i n q u i e ̀ m e ~ d e ~ s e s ~ p r i n c i p e s ~}{ }^{30}$. Ces références à Hayek, au demeurant souvent très vagues, nous paraissent toutefois occulter deux éléments qui rendent pourtant la pensée de l'auteur autrichien peu compatible avec certains projets transhumanistes. Le premier est la dénonciation d'un rationalisme constructiviste dont beaucoup d’idées transhumanistes ne paraissent pas dénuer. L’ordre spontanée chez Hayek n'est pas seulement un ordre qui, synchroniquement, n'a besoin d'aucune intervention volontaire centralisée pour fonctionner. C’est aussi, diachroniquement, un ordre que personne n’a jamais vraiment voulu, et que nul n'était en mesure de faire advenir. Or, on trouve bien

\footnotetext{
${ }^{28}$ James Hughes, Citizen Cyborg, op. cit. p. 19-20. Il est intéressant de retrouver le même exemple sous la plume de Raymond Kurzweil : « Notre système digestif a en particulier été conçu pour une situation radicalement différente de la nôtre aujourd'hui. Pour la plupart de nos ancêtres, la probabilité de n’obtenir de la cueillette ou de la chasse que de maigres ressources alimentaires était très élevée. Il était donc logique pour notre corps de préserver la moindre calorie. Aujourd'hui, cette fonction est contre-productive. La désuétude de notre programme métabolique sous-tend l'épidémie contemporaine d'obésité et alimente nombre de pathologies dégénératives comme les maladies coronariennes ou le diabète de type II. » Raymond Kurzweil, « Human Body version 2.0 » (2003), cité dans Nicolas Le Dévédec, La société de l’amélioration, op. cit., p. 206.

${ }^{29}$ On retrouve étonnamment chez Michael Sandel une critique comparable. «Changer notre nature pour nous adapter au monde, et non l'inverse, est en réalité la forme la plus profonde d'aliénation. Elle nous détourne d'une réflexion critique sur le monde, et tue tout désir de progrès social et politique. Plutôt que d'employer nos compétences génétiques à redresser le "bois tordu" dans lequel l’homme a été taillé, nous devrions faire tout notre possible pour créer des dispositifs politiques et sociaux plus adaptés aux dons et aux limitations d'êtres humains imparfaits. " Michael J. Sandel, Contre la perfection. L'éthique à l'âge du génie génétique (2007), traduit de l'américain par Hélène Valance, Paris, Vrin, 2016, p. 70.

30 Max More, 2 The Pxtropian 2.5 », 1993 , [http://www.aleph.se/Trans/Cultural/Philosophy/princip.html]
} 
chez certains transhumaniste une ambition prométhéenne de substituer à l'évolution naturelle une forme de «sélection délibérée ${ }^{31}$ ", et d'ainsi faire des ordres spontanés que sont, chez Hayek, le marché, la société et même le cerveau humain, des ordres organisés dont l'origine deviendrait imputable à une intention, et le fonctionnement susceptible d'un contrôle. Hayek adresserait vraisemblablement à certaines des techniques applaudies par les transhumanistes une critique similaire à celle qu'il formulait à l'encontre des projets de « justice sociale ». On ne peut selon Hayek appliquer les prédicats «juste » et «injuste » qu’à des actions individuelles : «À strictement parler, seule la conduite humaine peut être appelée juste ou injuste. [...] Un fait en lui-même, ou un état de choses que personne ne peut changer, peut être bon ou mauvais, mais non pas juste ou injuste [...] Ce n'est que si nous entendons blâmer un créateur personnel que cela peut avoir un sens de qualifier d'injuste le fait que quelqu'un soit né avec une malformation physique, ou qu'il ait contracté une maladie ou perdu un être aimé. La nature ne peut être juste ni injuste ${ }^{32}$. » Parler de justice ou d'injustice n’a ainsi de sens que si quelqu'un a accompli volontairement une action et qu'il peut en être tenu pour responsable. L'exigence de justice sociale revient alors ou bien à croire en l'existence d'une force anthropomorphique, ou bien à vouloir que l’ordre spontané de société se transforme en une organisation capable de répartir les richesses conformément à une idée déterminée de justice. Hayek souscrirait ainsi sans doute à la critique formulée par Michael Sandel à l'encontre de l'augmentation génétique, dont le véritable problème serait «l'expansion, non l'effacement, de la responsabilité. Tandis que l’humilité disparaît, la responsabilité prend des proportions effrayantes. Nous attribuons moins au hasard et plus au choix. Les parents ont la responsabilité de choisir, ou de ne pas parvenir à choisir, les bonnes caractéristiques génétiques pour leurs enfants. Les sportifs ont la responsabilité d'acquérir, ou de ne pas parvenir à acquérir, les talents qui aideront leur équipe à gagner ${ }^{33}$. " Le transhumanisme, parce qu'il appelle de ses vœux une intervention volontaire plus grande pour conjurer les hasards de la nature, étendrait imprudemment le champ de la juridiction humaine.

Un deuxième aspect de la théorie hayékienne qui semble échapper aux transhumanistes est qu'elle repose sur le constat de la finitude humaine qu'illustre parfaitement notre ignorance indépassable. Hayek écrit ainsi que «la justification de la liberté individuelle se

\footnotetext{
${ }^{31}$ L'expression est de John Harris. « Ce nouveau processus évolutif remplacera la sélection naturelle par une sélection délibérée, l'évolution darwinienne par une "évolution de l'augmentation". » John Harris, Enhancing Evolution: The Ethical Case for Making People Better, Princeton, Princeton University Press, 2010, p. 4.

${ }^{32}$ Friedrich A. Hayek, Droit, législation et liberté, Tome II, Le mirage de la justice sociale (1976), traduit de l'anglais par Raoul Audouin, Paris, PUF, « Quadrige », 1981, p. 37-38

${ }^{33}$ Michael J. Sandel, Contre la perfection, op. cit., p. 64.
} 
fonde principalement sur le constat de notre inévitable ignorance ${ }^{34} »:$ « La certitude ne peut être obtenue dans les affaires humaines ; c'est pourquoi si nous voulons faire le meilleur usage possible du peu de connaissances que nous avons, il nous faut adhérer à des règles dont l'expérience a montré qu'elles sont globalement satisfaisantes ; encore que nous ne sachions pas, dans chaque cas concret, quelle conséquence découlera de notre obéissance auxdites règles $^{35}$. » Ce sur quoi Hayek entendait fonder sa défense de la liberté est donc précisément ce à quoi les transhumanistes prétendent pouvoir remédier. Au fond, seuls des cyborgs, des zombis ou des avatars pourraient vivre ensemble sans un régime de liberté. À l'exception de sa version randienne, le libertarianisme destine ainsi les règles qu'il défend aux hommes tels qu'ils sont, et non tels qu'il aurait aimé qu'ils fussent. Comme l'écrit Murray Rothbard, « les libertariens ne considèrent pas que l'avènement de la pure société libre de leurs rêves s'accompagnera de la venue d'un homme libertarien nouveau magiquement transformé36 ». De la même manière Jan Narveson affirme que les institutions politiques doivent être conçues « pour les gens tels qu'ils sont, et non tels qu'ils pourraient être. [...] Pour les gens tels qu'ils sont, il n’y a pas de substituts à la propriété et à la liberté individuelles ${ }^{37}$. » Le transhumanisme rendrait ainsi obsolètes toutes les théories politiques ${ }^{38}$ destinées à l'homme tel qu'il est, fini et non augmenté. L'avènement du posthumain rendrait possible le déploiement du totalitarisme. Pour résumer, le libertarianisme, à l'exception notable de la version qu'en a proposée Ayn Rand, se focalise sur les règles sociales qui assureront la coexistence pacifique entre des êtres inévitablement imparfaits à la poursuite de leur propre conception de la vie bonne, et refuse de traiter la question de savoir en quoi consisterait leur accomplissement vertueux. Le libertarianisme s’intéresse davantage aux règles qu'aux comportements qu'elles régissent, davantage aux libertés qu'à ce qu'elles permettent d'accomplir, davantage à la justice des règles qu'à la moralité des conduites qu'elles

${ }^{34}$ Friedrich A. Hayek, La Constitution de la liberté (1960), traduit de l'anglais par Raoul Audouin et Jacques Garello, Paris, Litec, 1994, p. 30.

${ }^{35}$ Ibid., p. 31.

${ }^{36}$ Murray Rothbard, For a new liberty : The Libertarian manifesto (1973), Auburn, Mises Institute, 2002, p. 239.

${ }^{37}$ Jan Narveson, You and the State (2008), Lanham, Rowman \& Littlefield Publishers, 2008, p. 196-197.

${ }^{38}$ On pourrait en effet dire la même chose des autres théories politiques. La théorie de la justice de Rawls repose par exemple en grande partie sur l'impossibilité de neutraliser les effets de la loterie naturelle. Le transhumanisme, s'il remplit sa promesse d'égaliser les biens premiers naturels, rendrait en même temps inutile le principe de différence, que Rawls introduit uniquement dans le but de rendre supportables les hasards irréductibles de la naissance. Ce point est entraperçu par Michael Sandel. «Si le génie génétique nous permettait de dépasser la loterie génétique, de remplacer le hasard par le choix, alors le caractère donné des compétences et réussites humaines reculerait, et avec lui, peut-être, notre capacité à nous voir comme partageant un sort commun. Ceux qui réussissent seraient encore plus susceptibles qu'ils ne le sont maintenant de se considérer comme les fils de leurs œuvres, des individus auto-suffisants et par là seuls responsables de leur propre succès. Ceux qui, en revanche, se trouvent aux échelons les plus bas de la société seraient considérés non plus comme désavantagés, et donc méritant des mesures de compensation, mais comme des individus simplement inadaptés, méritant donc une réparation eugénique. » Michael J. Sandel, Contre la perfection, op. cit., p. 67. 
n’interdisent pas. Le transhumanisme présente une ambition opposée : il n’envisage que les moyens d'un épanouissement individuel, et néglige ainsi délibérément les conditions sociales d'une amélioration.

On aimerait enfin, pour clore cet examen des emprunts peu tatillons faits par les transhumanistes aux libertariens, dire un mot de la pensée de Nozick. Un passage parfois cité de Anarchie, État et Utopie envisage la possibilité d'un « "supermarché génétique" répondant aux spécifications individuelles (dans la limite de certaines règles morales) de parents éventuels. [...] Ce système de supermarché possède la grande vertu de n’impliquer aucune décision centralisée établissant le (ou les) type(s) humain(es) futur(s) ${ }^{39}$. " Cet extrait, tiré d'une simple note de bas de page, ne vaut pourtant pas quitus aux théories transhumanistes. Premièrement, Nozick dit simplement qu'un tel système serait préférable à un eugénisme étatisé aux accents totalitaires. Deuxièmement, Nozick envisage explicitement des restrictions morales, bien qu'il se garde de les préciser. On soulignera pour conclure que la célèbre machine à expériences, imaginée par Nozick pour critiquer l'utilitarisme hédoniste, condamnerait aussi bon nombre de projets transhumanistes. "Supposez, demande Nozick, qu'il existe une machine à expérience qui soit en mesure de vous faire vivre n’importe quelle expérience que vous souhaitez. Des neuropsychologues excellant dans la duperie pourraient stimuler votre cerveau de telle sorte que vous croiriez et sentiriez que vous êtes en train d'écrire un grand roman, de vous lier d'amitié, ou de lire un livre intéressant. Tout ce tempslà, vous seriez en train de flotter dans un réservoir, des électrodes fixées à votre crâne. Faudrait-il que vous branchiez cette machine à vie, établissant d'avance un programme des expériences de votre existence ${ }^{40}$ ? » Nozick répond par la négative, en prenant notamment appui sur l'exemple des drogues psychoactives souvent plébiscitées par les transhumanistes, et en argumentant que peu de gens accepteraient de laisser à des machines la tâche de « vivre nos vies pour nous ${ }^{41}$ » et se priver ainsi d’un "contact véritable avec une réalité plus profonde ${ }^{42} »$.

On retiendra ainsi qu'il n’existe pas d'implication réciproque entre les deux théories. Le transhumanisme peut ne pas être libertarien; le libertarianisme peut ne pas être transhumaniste. Si le libertarianisme n’offre pas une théorie morale venant justifier le transhumanisme, force est cependant de reconnaître qu'il ne constitue pas non plus une

\footnotetext{
${ }^{39}$ Robert Nozick, Anarchie, État et Utopie (1974), traduit de l'anglais par. Evelyne D'Auzac de Lamartine, Paris, PUF, 1988, p. 384.

${ }^{40}$ Ibid., p. 64.

${ }^{41}$ Ibid., p. 66.

${ }^{42}$ Ibid., p. 65.
} 
théorie politique qui viendrait l'exclure en tant que mode de vie. La question qui se pose alors est de savoir si, en ne l'interdisant pas, le libertarianisme n’offre pas au transhumanisme les moyens de se développer, de prospérer, voire de s’imposer.

\section{Le libertarianisme, cheval de Troie du transhumanisme}

Le silence des libertariens sur le transhumaniste nous informe peut-être sur leur réticence à le soutenir, mais en dit également long sur leur incapacité à le répudier. Il nous faut ainsi constater que, si le libertarianisme n'offre au fond aucune justification théorique au transhumanisme, il ne lui oppose aucune résistance. Parce qu’il s’interrompt au seuil de la morale individuelle, il se révèle incapable de préserver nos sociétés de l'expansion du transhumanisme. En bref, le libertarianisme ne peut interdire ce que le transhumanisme promeut. C’est cette connivence que viennent parfois dénoncer les critiques communautariennes et conservatrices du transhumanisme. Parce que la pensée libérale contemporaine, et a fortiori la philosophie libertarienne, défend l’idée d'un droit indifférent à la morale, et destine ainsi ses principes juridiques à des coquilles vides, à des atomes déracinés plus proches de robots que d'humains, elle encouragerait ce qu'elle ne prohibe pas, et se révèlerait extrêmement hospitalière au transhumanisme. Parmi les diverses conceptions de la vie bonne, une politique du bien commun permettrait au moins de faire le tri. C'est par exemple le fond de l'argumentaire de Francis Fukuyama, qui dénonce l’incapacité de toute doctrine déontologique à interdire ce qui semble pourtant moralement condamnable ${ }^{43}$. «Les théories déontologiques finissent par élever l'autonomie morale individuelle au rang de suprême bien humain. Elles proposent aux individus le marché suivant : ni les philosophes ni la société - sous la forme d’un État libéral - ne vous indiqueront comment vivre votre vie, mais vous laisseront décider. Tout ce qu'ils feront sera d'établir certaines règles de procédure afin d'assurer que le plan de vie vous avez choisi n’interfère pas avec ceux de vos concitoyens. [...] Le droit de choisir est la seule chose que les théories déontologiques protègent vraiment ${ }^{44}$. » On peut alors noter une forte analogie entre les deux théories : de même que le transhumanisme se voudrait apolitique (et susceptible d'être tour à tour - ou tout à la fois - libertarien, libéral, démocrate ou marxiste) le libertarianisme se prétend amoral (et

\footnotetext{
${ }^{43}$ Il est intéressant de noter ici que Nozick, dans le passage que nous avons cité plus haut, ne cherche pas au fond à justifier l'idée d’un supermarché génétique, mais plutôt à voir si nous avons des raisons de le répudier.

${ }^{44}$ Francis Fukuyama, La fin de l'homme. Les conséquences de la révolution biotechnique, traduit de l'américain par Denis-Armand Canal, Paris, La table ronde, 2002, p. 186.
} 
acceptable pour n'importe quelle conception du bien). Ce faisant, le libertarianisme n’offre aucune bonne raison d’interdire les techniques amélioratives défendues par le transhumanisme.

Mais les critiques communautariennes et conservatrices portent un peu plus loin. Les théories politiques qui donneraient la priorité au juste sur le bien n’auraient pas seulement l’inconvénient de ne pouvoir interdire des visions fortement condamnables du bien : elles les encourageraient subrepticement. À l'instar des communautariens, Fukuyama en vient ainsi à dénoncer la fausse neutralité du libéralisme: "L’éthique fondée sur des principes déontologiques n'est pas véritablement neutre parmi les plans de vie : elle favorise les plus individualistes qui prédominent, dans les sociétés libérales, sur les plus communautaires ; or ces dernières peuvent être tout autant satisfaisante sur le plan humain ${ }^{45}$. » Bien qu'elle confonde abusivement libéralisme et doctrine déontologique, cette critique peut s'étendre à toutes les théories prônant une séparation du droit et de la morale. Toutefois, elle ne permet pas à elle seule d'expliquer l'affinité élective empiriquement observable entre le libertarianisme et le transhumanisme ${ }^{46}$. On peut alors tenter de voir si, parmi les doctrines « libérales », le libertarianisme n’est pas celle qui encourage le plus (ou décourage le moins) le transhumanisme. Parce que le libertarianisme livrerait les individus à une concurrence impitoyable où seuls les plus performants seraient récompensés, il semble en effet inciter chacun à recourir aux techniques d'amélioration offertes par les apôtres du transhumanisme. Les libertariens revendiqueraient ainsi une neutralité biaisée, offrant théoriquement à chacun la liberté de ne pas s'augmenter, mais ne laissant en pratique à personne la possibilité de réussir sa vie sans consentir à s’améliorer. Comme l'écrit Sandel, en effet, «l’impulsion prométhéenne est contagieuse ${ }^{47}$ ", et il est difficile d’imaginer qu'une compétition sportive puisse confronter des humains non modifiés à des cyborgs suréquipés, ou encore que certains candidats à un concours d'enseignement s'obstinent à bachoter un programme que d'autres auraient téléchargé la veille dans leur cerveau. Kevin Warwick, professeur de cybernétique à l’Université de Coventry, connu pour s’être greffé dans l'avant-bras des électrodes lui permettant de commander à distance son ordinateur, reconnaît ainsi que « ceux qui décideront

\footnotetext{
${ }^{45}$ Ibid., p. 188.

${ }^{46}$ Étant donné le contexte de la vie politique américaine, les transhumanistes auraient trouvé plus d'avantage à s’allier avec la gauche progressiste démocrate.

${ }^{47}$ Michael J. Sandel, Contre la perfection, op. cit., p. 65.
} 
de rester humains et refuseront de s'améliorer auront un sérieux handicap. Ils constitueront une sous-espèce et formeront les chimpanzés du futur ${ }^{48}$. »

Le libertarianisme entretient donc avec le transhumanisme un rapport hautement paradoxal. Dans le contexte où l’État manifeste quelques réticences à autoriser les techniques amélioratives les plus audacieuses, les libertariens livrent tout d'abord stratégiquement aux transhumanistes de précieuses munitions théoriques (notamment les principes de propriété sur soi et du libre consentement), délégitimant toute intrusion du politique dans la vie privée des individus. En retour, le développement des technologies encouragé par le transhumanisme donnerait du crédit aux théories libertariennes qui dénoncent l'obsolescence de l'Etat ${ }^{49}$. Une fois appliqué, même partiellement, le libertarianisme créerait ensuite, par la concurrence exacerbée qu'il instaurerait, un besoin que le transhumanisme voudrait pouvoir mieux satisfaire, en offrant aux individus les moyens d'être toujours plus compétitifs. Mais, on l'a vu, cette complicité de circonstances ne saurait vraiment durer. Un transhumanisme non empêché, et l'avènement sans entraves de tous ses possibles, éradiquerait deux conditions qui viennent justifier le libertarianisme, et lui donner sa raison d’être. En dépit des dénégations appuyés des auteurs transhumanistes, la venue du posthumain anéantirait premièrement tout pluralisme des valeurs, et conduirait irrésistiblement à une forme de monisme de facto, sinon de jure. Les transhumanistes peinent à convaincre que le libre choix laissé aux individus (de s'augmenter ou non) serait la garantie d'une préservation du pluralisme et de la biodiversité. Le transhumanisme réalisé, ou posthumanisme, sécrèterait deuxièmement des hommes presque parfaits, quasi-omniscients, peut-être même immortels et entre lesquels il serait tout bonnement vain de faire régner la liberté ${ }^{50}$. Si le libertarianisme peut, provisoirement, constituer la théorie politique susceptible de hâter l'avènement de l'homme augmenté, s'il peut à la rigueur régir les rapports sociaux des hommes en voie d'amélioration, il paraît fortement inadapté aux posthumains. Pour paraphraser Madison («Si les hommes étaient des anges, ils n’auraient pas besoin du gouvernement »), on peut ainsi considérer que si les

\footnotetext{
${ }^{48}$ Kevin Warwick, cité dans Nicolas Le Dévédec, La société de l'amélioration, op. cit., p. 207.

${ }^{49}$ Cette alliance stratégique se trouve explicitement exposée dans le dernier livre de l'expert du Cato Institute Jason Kuznicki (Technology and the End of Authority, op. cit.) qui considère que les nouvelles technologies «semblent susceptibles de remettre en cause une part importante de la manière que nous avons de justifier l'Etat » (p. 235). On retrouve une argumentation similaire chez Alex Tabarrok et Tyler Cowen (« The End of Asymmetric Information », Cato Unbound, 2015) qui soutiennent que la réduction, par les nouvelles technologies, de l'asymétrie informationnelle rendrait obsolète la plupart des régulations étatiques.

${ }^{50}$ Hayek écrit par exemple que « si à un moment donné quelqu'un pouvait connaître la somme totale de tous les faits particuliers dispersés parmi les millions ou les milliards de personnes vivant à un moment donné, il devrait être en mesure d'apporter aux efforts humains de production un ordre plus efficace que ne le fait le marché. » Friedrich A. Hayek, Nouveaux essais de philosophie, de science politique et d'économie (1978), traduit de l'anglais par Christophe Piton, Paris, Les Belles Lettres, 2008, p. 38.
} 
hommes étaient des posthumains, ils n’auraient pas besoin de libertarianisme. Le libertarianisme serait alors au transhumanisme ce que la dictature du prolétariat était au communisme: un stade transitoire, ou un moyen de faire advenir ce qui le dépassera et consacrera sa désuétude. Parmi les conditions sociales d'existence des individus, le transhumanisme n'envisage que celles (possiblement libertariennes) qui lui permettront de faire advenir l’homme augmenté, mais néglige très souvent celles qui devront régir l'existence collective des posthumains. Leur vue morale porte loin. Mais leur vue sociale n'est qu'à court terme. On peut alors considérer que le transhumanisme ne se contenterait pas de rendre inutile le libertarianisme. Il ouvrirait potentiellement la voie à son contraire totalitaire. Cette dialectique, funeste pour le libertarianisme, illustre à elle-seule la contradiction logique qui semble miner la doctrine, condamnée à rejeter par principe les conditions politiques de son maintien, et à ne pouvoir proscrire les éléments qui, programmant son obsolescence, précipitent sa propre destruction.

\section{Notice biobibliographique}

Sébastien Caré est maître de conférences en science politique à l'Université Rennes 1 et chercheur à l'Institut du Droit Public et de la Science Politique (IDPSP). Ses travaux portent sur l'histoire des idées politiques, et plus particulièrement sur les théories libérales contemporaines. Il a notamment publié La pensée libertarienne: Genèse, fondements et horizons d'une utopie libérale (Paris, PUF, 2009) et Les libertariens aux Etats-Unis: Sociologie d'un mouvement asocial (Rennes, PUR, 2010).

Sébastien Caré is Associate Professor in Political science at Rennes 1 University. He works on history of contemporary political ideas, with particular interest in classical liberal theories. He is the author of La pensée libertarienne: Genèse, fondements et horizons d'une utopie libérale (Paris, PUF, 2009) and Les libertariens aux Etats-Unis : Sociologie d'un mouvement asocial (Rennes, PUR, 2010).

\section{Résumé}

\section{La route (libertarienne) de la servitude (transhumaniste)}


Cet article interroge la pertinence théorique des liens historiquement constitués entre le libertarianisme et le transhumanisme. Il s'attache tout d'abord à démontrer qu'il n’y a entre les deux théories aucune implication réciproque. D’un côté, le transhumanisme peut ne pas être libertarien : l'avènement d'un homme nouveau, augmenté par la technique, ne passe pas nécessairement par la défense d’un État minimal. D’un autre côté, le libertarianisme peut ne pas être transhumaniste : le rejet d'un État étendant ses prérogatives au-delà de la simple protection des personnes ne suppose pas une amélioration fondamentale de la condition humaine et la promotion de toutes les technologies susceptibles d'y conduire. Cet article analyse ensuite les rapports entre transhumanisme et libertarianisme en envisageant la compossibilité de leurs réalisations respectives. Si le libertarianisme ne justifie aucunement le transhumanisme, il ne le proscrit pas, et sa concrétisation pourrait même l'encourager en instaurant une concurrence exacerbée et en créant ainsi faisant un besoin de performance que les technologies amélioratives voudraient pouvoir satisfaire. En retour, la réalisation du transhumanisme, et l'avènement de l'homme augmenté, consacrerait cependant l'obsolescence d'une théorie libertarienne originellement destinée à des êtres très imparfaits, et ouvrirait la possibilité de son contraire totalitaire.

\section{The (libertarian) road to (transhumanist) serfdom}

This article questions the theoretical relevance of the historical links between libertarianism and transhumanism. First, it seeks to demonstrate that there is no mutual implication between the two theories. On the one hand, transhumanism is not necessarily libertarian: the advent of a new man, enhanced by technology, does not require the defence of a minimal state. On the other hand, libertarianism may not be transhumanist: the rejection of a State extending its prerogatives beyond the mere protection of individuals does not imply a fundamental improvement of the human condition and the promotion of all the technologies that could lead to it. This article then analyses the relationship between transhumanism and libertarianism by considering the compossibility of their respective achievements. While libertarianism in no way justifies transhumanism, it does not prohibit it, and its realization could even encourage it by introducing increased competition and thus creating a need for performance that improved technologies would seek to satisfy. However, the realization of transhumanism, and the advent of the enhanced man, would consecrate in return the obsolescence of a libertarian theory originally intended for very imperfect beings, and would open the possibility of its totalitarian opposite. 